Теорія Ймовір. та Матем. Статист. Вип. 76, 2007
Theor. Probability and Math. Statist.

No. 76, 2008, Pages 49-57

S 0094-9000(08)00731-X

Article electronically published on July 14, 2008

\title{
OVERSHOOT FUNCTIONALS FOR ALMOST SEMICONTINUOUS PROCESSES DEFINED ON A MARKOV CHAIN
}

UDC 519.21

\author{
E. V. KARNAUKH
}

\begin{abstract}
The distributions of overshoot functionals are considered in the paper for almost semicontinuous processes defined on a finite irreducible Markov chain.
\end{abstract}

\section{INTRODUCTION}

The distributions of extremal values and overshoot functionals for semicontinuous processes (that is, for those processes that cross either a positive or a negative barrier in a continuous way) defined on a Markov chain are considered by many authors (see, for example, [1]-3]). The distributions of extremal values are considered in the paper [4 for almost semicontinuous processes (that is, for those processes that cross either a positive or a negative barrier by means of exponential jumps only). Under some assumptions, these processes can be viewed as surplus risk processes with random premiums in a Markov environment. The distributions of some overshoot functionals are studied in this paper for lower almost semicontinuous processes defined on a Markov chain.

\section{MAIN PART}

Consider a two dimensional Markov process

$$
Z(t)=\{\xi(t), x(t)\}, \quad t \geq 0,
$$

where $x(t)$ is a finite irreducible aperiodic Markov chain whose phase space is

$$
E^{\prime}=\{1, \ldots, m\}
$$

and whose matrix of transient probabilities is given by

$$
\mathbf{P}(t)=e^{t \mathbf{Q}}, \quad t \geq 0, \quad \mathbf{Q}=\mathbf{N}(\mathbf{P}-\mathbf{I}) .
$$

Here

$$
\mathbf{N}=\left\|\delta_{k r} \nu_{k}\right\|_{k, r=1}^{m}
$$

$\nu_{k}$ are the parameters of the exponential random variables $\zeta_{k}$ (meaning the sojourn times of $x(t)$ at states $k), \mathbf{P}=\left\|p_{k r}\right\|$ is the matrix of transient probabilities of the embedded chain, and $\boldsymbol{\pi}=\left(\pi_{1}, \ldots, \pi_{m}\right)$ is the stationary distribution. The process $\xi(t)$ is homogeneous with conditionally independent increments given that the values of $x(t)$ are fixed (see [1]).

The evolution of the process $Z(t)$ is described by the matrix characteristic function

$$
\boldsymbol{\Phi}_{t}(\alpha)=\left\|\mathrm{E}\left[e^{\imath \alpha(\xi(t+u)-\xi(u))}, x(t+u)=r / x(u)=k\right]\right\|, \quad u \geq 0,
$$

2000 Mathematics Subject Classification. Primary 60G50, 60J70; Secondary 60K10, 60K15.

Key words and phrases. Overshoot functionals, almost semicontinuous processes, ruin probability. 
which can be represented in the following form:

$$
\boldsymbol{\Phi}_{t}(\alpha)=\mathbf{E} e^{\imath \alpha \xi(t)}=e^{t \boldsymbol{\Psi}(\alpha)}, \quad \boldsymbol{\Psi}(0)=\mathbf{Q} .
$$

In what follows we study the processes with the cumulant

$$
\mathbf{\Psi}(\alpha)=\int_{0}^{\infty}\left(e^{\imath \alpha x}-1\right) d \mathbf{K}_{0}(x)+\mathbf{\Lambda} \mathbf{F}_{0}(0)\left(\mathbf{C}(\mathbf{C}+\imath \alpha \mathbf{I})^{-1}-\mathbf{I}\right)+\mathbf{Q},
$$

where $d \mathbf{K}_{0}(x)=\mathbf{N} d \mathbf{F}(x)+\mathbf{\Pi}(d x)$,

$$
\mathbf{F}(x)=\left\|\mathrm{P}\left\{\chi_{k r}<x ; x\left(\zeta_{1}\right)=r / x(0)=k\right\}\right\|,
$$

$\chi_{k r}$ are the heights of jumps of $\xi(t)$ at the moments when $x(t)$ jumps from the state $k$ to the state $r$,

$$
\Pi(d x)=\boldsymbol{\Lambda} d \mathbf{F}_{0}(x), \quad \mathbf{F}_{0}(x)=\left\|\delta_{k r} F_{k}^{0}(x)\right\|,
$$

$F_{k}^{0}(x)$ are the distribution functions of the heights of jumps of $\xi(t)$ given $x(t)=k$, $\boldsymbol{\Lambda}=\left\|\delta_{k r} \lambda_{k}\right\|$, and where $\lambda_{k}$ are the parameters of the exponential random variables $\zeta_{k}^{\prime}$ (meaning the time between two successive jumps of $\xi(t)$ given $x(t)=k$ ). Let $\mathbf{C}=\left\|\delta_{k r} c_{k}\right\|$, where $c_{k}$ are the parameters of the exponential negative jumps of $\xi(t)$ given $x(t)=k$. A process $Z(t)$ with a cumulant of this kind is called a lower almost semicontinuous process (this definition is introduced in [4]).

Denote by $\theta_{s}$ an exponential random variable whose parameter is $s>0$ (that is, $\mathrm{P}\left\{\theta_{s}>t\right\}=e^{-s t}$ for $\left.t \geq 0\right)$ and assume that $\theta_{s}$ is independent of $Z(t)$. Then the characteristic function of $\xi\left(\theta_{s}\right)$ can be written as follows:

$$
\mathbf{\Phi}(s, \alpha)=\mathbf{E} e^{\imath \alpha \xi\left(\theta_{s}\right)}=s \int_{0}^{\infty} e^{-s t} \mathbf{\Phi}_{t}(\alpha) d t=s(s \mathbf{I}-\mathbf{\Psi}(\alpha))^{-1} .
$$

We introduce the main functionals of interest:

$$
\begin{array}{cc}
\xi^{ \pm}(t)=\sup _{0 \leq u \leq t}(\inf ) \xi(u), & \xi^{ \pm}=\sup _{0 \leq u \leq \infty}(\inf ) \xi(u) ; \\
\bar{\xi}(t)=\xi(t)-\xi^{+}(t), & \check{\xi}(t)=\xi^{-}(t)-\xi(t), \\
\tau^{+}(x)=\inf \{t: \xi(t)>x\}, & \gamma^{+}(x)=\xi\left(\tau^{+}(x)\right)-x, \quad x \geq 0 . \\
\gamma_{+}(x)=x-\xi\left(\tau^{+}(x)-0\right), & \gamma_{x}^{+}=\gamma^{+}(x)+\gamma_{+}(x),
\end{array}
$$

The distributions of the functionals $\xi^{ \pm}\left(\theta_{s}\right), \bar{\xi}\left(\theta_{s}\right)$, and $\check{\xi}\left(\theta_{s}\right)$ are obtained in 4 . The aim of this paper is to obtain explicitly the joint moment generating functions of overshoot functionals for lower almost semicontinuous processes and moment generating functions of the random vectors $\left\{\tau^{+}(x), \gamma^{+}(x)\right\},\left\{\tau^{+}(x), \gamma_{+}(x)\right\}$, and $\left\{\tau^{+}(x), \gamma_{x}^{+}\right\}$.

Put

$$
\begin{gathered}
\mathbf{V}(s, x, u, v, \mu)=\mathbf{E}\left[e^{-s \tau^{+}(x)-u \gamma^{+}(x)-v \gamma_{+}(x)-\mu \gamma_{x}^{+}}, \tau^{+}(x)<\infty\right], \\
\mathbf{W}(x, u, v, \mu)=\int_{x}^{\infty} e^{(u-v) x-(u+\mu) z} d \mathbf{K}_{0}(z), \quad \overline{\mathbf{K}}_{0}(x)=\mathbf{W}(x, 0,0,0), \\
\mathbf{P}_{s}=s \int_{0}^{\infty} e^{-s t} \mathbf{P}(t) d t=s(s \mathbf{I}-\mathbf{Q})^{-1}, \\
\mathbf{P}_{+}(s, x)=\mathbf{P}\left\{\xi^{+}\left(\theta_{s}\right)<x\right\}, \quad x>0, \quad \mathbf{P}^{-}(s, x)=\mathbf{P}\left\{\bar{\xi}\left(\theta_{s}\right)<x\right\}, \quad x<0, \\
\widetilde{\mathbf{P}}^{0}(s)=\mathbf{P}\left\{\xi\left(\theta_{s}\right)=0\right\}, \quad \mathbf{p}_{ \pm}(s)=\mathbf{P}\left\{\xi^{ \pm}\left(\theta_{s}\right)=0\right\}, \quad \check{\mathbf{R}}_{-}(s)=\mathbf{P}_{s}^{-1} \check{\mathbf{p}}_{-}(s), \\
\check{\mathbf{p}}_{-}(s)=\mathbf{P}\left\{\bar{\xi}\left(\theta_{s}\right)=0\right\}, \quad \check{\mathbf{q}}_{-}(s)=\mathbf{P}_{s}-\check{\mathbf{p}}_{-}(s), \quad \check{\mathbf{R}}_{c}(s)=\check{\mathbf{R}}_{-}(s) \mathbf{C}, \\
\mathbf{G}_{+}(s, x, u, v, \mu)=\int_{-\infty}^{0} d \mathbf{P}^{-}(s, y) \mathbf{W}(x-y, u, v, \mu) .
\end{gathered}
$$


Lemma 1. Consider a process $Z(t)$ with the cumulant of the form (1). Then

$$
s \mathbf{V}(s, x, u, v, \mu)=\int_{0}^{x} d \mathbf{P}_{+}(s, y) \mathbf{P}_{s}^{-1} \mathbf{G}_{+}(s, x-y, u, v, \mu), \quad x>0,
$$

where

$$
\begin{aligned}
& \mathbf{G}_{+}(s, x, u, v, \mu) \\
&=\check{\mathbf{p}}_{-}(s) \int_{x}^{\infty} e^{(u-v) x-(u+\mu) z} d \mathbf{K}_{0}(z) \\
&-\check{\mathbf{p}}_{-}(s) \mathbf{C}\left(\mathbf{P}_{s}^{-1} \check{\mathbf{p}}_{-}(s) \mathbf{C}-(u-v) \mathbf{I}\right)^{-1} e^{-(v+\mu) x} \\
& \quad \times \int_{0}^{\infty}\left[(u+\mu) e^{-(u+\mu) z}\right. \\
& \quad-\left(\mathbf{P}_{s}^{-1} \check{\mathbf{p}}_{-}(s) \mathbf{C}+(\mu+v) \mathbf{I}\right) e^{\left.-\left(\mathbf{P}_{s}^{-1} \check{\mathbf{p}}_{-}(s) \mathbf{C}+(v+\mu) \mathbf{I}\right) z\right]} \\
& \quad \times \mathbf{P}_{s}^{-1} \check{\mathbf{q}}_{-}(s) \overline{\mathbf{K}}_{0}(x+z) d z
\end{aligned}
$$

for $u-v \notin \sigma\left(\mathbf{P}_{s}^{-1} \check{\mathbf{p}}_{-}(s) \mathbf{C}\right)$. Here the symbol $\sigma(A)$ stands for the spectrum of a matrix $A$.

Proof. Since we assume the almost semicontinuity, relation (3) follows from 1, Corollary 3.4]. According to [4] (see Remark 1 therein) the distribution of $\bar{\xi}\left(\theta_{s}\right)$ is given by

Then

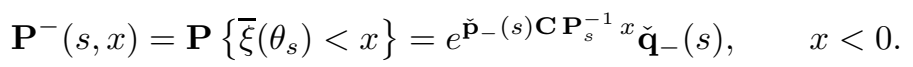

$$
\begin{aligned}
\mathbf{G}_{+}(s, x, u, v, \mu)= & \int_{-\infty}^{0} d \mathbf{P}^{-}(s, y) \mathbf{W}(x-y, u, v, \mu) \\
= & \check{\mathbf{p}}_{-}(s) \mathbf{W}(x, u, v, \mu) \\
& +\check{\mathbf{p}}_{-}(s) \mathbf{C} \int_{x}^{\infty} e^{\mathbf{P}_{s}^{-1} \check{\mathbf{p}}_{-}(s) \mathbf{C}(x-y)} \mathbf{P}_{s}^{-1} \check{\mathbf{q}}_{-}(s) \mathbf{W}(y, u, v, \mu) d y .
\end{aligned}
$$

By the definition of the function $\mathbf{W}(y, u, v, \mu)$,

$$
\begin{aligned}
\int_{x}^{\infty} & e^{\mathbf{P}_{s}^{-1} \check{\mathbf{p}}_{-}(s) \mathbf{C}(x-y)} \mathbf{P}_{s}^{-1} \check{\mathbf{q}}_{-}(s) \mathbf{W}(y, u, v, \mu) d y \\
= & -\left(\mathbf{P}_{s}^{-1} \check{\mathbf{p}}_{-}(s) \mathbf{C}-(u-v) \mathbf{I}\right)^{-1} e^{-(v+\mu) x} \\
& \times\left[\left(\mathbf{P}_{s}^{-1} \check{\mathbf{p}}_{-}(s) \mathbf{C}+(\mu+v) \mathbf{I}\right) \int_{0}^{\infty} e^{-\left(\mathbf{P}_{s}^{-1} \check{\mathbf{p}}_{-}(s) \mathbf{C}+(v+\mu) \mathbf{I}\right) z} \mathbf{P}_{s}^{-1} \check{\mathbf{q}}_{-}(s) \overline{\mathbf{K}}_{0}(x+z) d z\right. \\
& \left.\quad-(u+\mu) \int_{0}^{\infty} e^{-(u+\mu) z} \mathbf{P}_{s}^{-1} \check{\mathbf{q}}_{-}(s) \overline{\mathbf{K}}_{0}(x+z) d z\right] .
\end{aligned}
$$

Now equality (4) follows from (5).

Note that

$$
\lim _{x \rightarrow-\infty} \mathbf{P}^{-}(s, x)=\mathbf{P}\left\{\bar{\xi}\left(\theta_{s}\right)<-\infty\right\}=0 .
$$

Thus the representation

$$
\begin{aligned}
\mathbf{P}^{-}(s, x) & =e^{\check{\mathbf{p}}-(s) \mathbf{C} \mathbf{P}_{s}^{-1} x} \mathbf{q}^{-}(s)=\mathbf{P}_{s} \mathbf{P}_{s}^{-1} e^{\check{\mathbf{P}}_{-}(s) \mathbf{C} \mathbf{P}_{s}^{-1}} \mathbf{P}_{s} \mathbf{P}_{s}^{-1} \check{\mathbf{q}}-(s) \\
& =\mathbf{P}_{s} e^{\check{\mathbf{R}}_{c}(s) x}\left(\mathbf{I}-\check{\mathbf{R}}_{-}(s)\right)
\end{aligned}
$$

implies that the spectrum $\sigma\left(\check{\mathbf{R}}_{c}(s)\right)$ of the matrix $\check{\mathbf{R}}_{c}(s)$ consists of positive elements.

Let

$$
\gamma_{1}(x)=\gamma^{+}(x), \quad \gamma_{2}(x)=\gamma_{+}(x), \quad \gamma_{3}(x)=\gamma_{x}^{+} .
$$


E. V. KARNAUKH

Substituting $v=\mu=0, u \notin \sigma\left(\check{\mathbf{R}}_{c}(s)\right), u=\mu=0$, and $v=u=0$ we derive from equality (3) that

$$
\begin{aligned}
& \mathbf{E}\left[e^{-s \tau^{+}(x)-u \gamma_{i}(x)}, \tau^{+}(x)<\infty\right]=s^{-1} \int_{0}^{x} d \mathbf{P}_{+}(s, y) \mathbf{P}_{s}^{-1} \mathbf{G}_{i}(s, x-y, u), \quad i=1,2,3, \\
& \mathbf{G}_{1}(s, x, u)= \check{\mathbf{p}}_{-}(s) \int_{x}^{\infty} e^{u(x-z)} d \mathbf{K}_{0}(z) \\
&-\check{\mathbf{p}}_{-}(s) \mathbf{C}\left(\check{\mathbf{R}}_{c}(s)-u \mathbf{I}\right)^{-1} \\
& \times \int_{0}^{\infty}\left[u e^{-u z}-\check{\mathbf{R}}_{c}(s) e^{-\check{\mathbf{R}}_{c}(s) z}\right] \mathbf{P}_{s}^{-1} \check{\mathbf{q}}_{-}(s) \overline{\mathbf{K}}_{0}(x+z) d z \\
& \mathbf{G}_{2}(s, x, v)=\check{\mathbf{p}}_{-}(s) e^{-v x} \overline{\mathbf{K}}_{0}(x)+\check{\mathbf{p}}_{-}(s) \mathbf{C} e^{-v x} \int_{0}^{\infty} e^{-\left(\check{\mathbf{R}}_{c}(s)+v \mathbf{I}\right) z} \mathbf{P}_{s}^{-1} \check{\mathbf{q}}_{-}(s) \overline{\mathbf{K}}_{0}(x+z) d z, \\
& \mathbf{G}_{3}(s, x, \mu)=\check{\mathbf{p}}_{-}(s) \int_{x}^{\infty} e^{-\mu z} d \mathbf{K}_{0}(z) \\
& \quad-e^{-\mu x} \int_{0}^{\infty}\left[\mu e^{-\mu z}-\left(\check{\mathbf{R}}_{c}(s)+\mu \mathbf{I}\right) e^{-\left(\check{\mathbf{R}}_{c}(s)+\mu \mathbf{I}\right) z}\right] \mathbf{P}_{s}^{-1} \check{\mathbf{q}}_{-}(s) \overline{\mathbf{K}}_{0}(x+z) d z .
\end{aligned}
$$

Inverting with respect to $u$ we obtain

$$
\begin{gathered}
\mathbf{E}\left[e^{-s \tau^{+}(x)}, \gamma_{i}(x) \in d z, \tau^{+}(x)<\infty\right]=s^{-1} \int_{0}^{x} d \mathbf{P}_{+}(s, y) \check{\mathbf{R}}_{-}(s) d_{z} \mathbf{g}_{i}^{*}(s, x-y, z), \\
d_{z} \mathbf{g}_{i}^{*}(s, x, z)=d_{z} \mathbf{w}_{i}^{*}(x, z)+\mathbf{C} \int_{x}^{\infty} e^{\check{\mathbf{R}}_{c}(s)(x-y)}\left(\mathbf{I}-\check{\mathbf{R}}_{-}(s)\right) d_{z} \mathbf{w}_{i}^{*}(y, z) d y, \\
d_{z} \mathbf{w}_{1}^{*}(x, z)=d_{z} \mathbf{K}_{0}(x+z), \quad d_{z} \mathbf{w}_{2}^{*}(x, z)=d_{z} I\{z>x\} \overline{\mathbf{K}}_{0}(x), \\
d_{z} \mathbf{w}_{3}^{*}(x, z)=I\{z \geq x\} d \mathbf{K}_{0}(z) .
\end{gathered}
$$

The case of $x=0$ is treated in the following result.

Theorem 1. Consider a process $Z(t)$ with the cumulant of the form (1). If $z>0$, then

$$
\begin{gathered}
\mathbf{E}\left[e^{-s \tau^{+}(0)}, \gamma^{+}(0)>z, \tau^{+}(0)<\infty\right] \\
=s^{-1} \widetilde{\mathbf{P}}^{0}(s)\left(\overline{\mathbf{K}}_{0}(z)+\mathbf{C} \int_{z}^{\infty} e^{(z-y) \check{\mathbf{R}}_{c}(s)} \mathbf{P}_{s}^{-1} \check{\mathbf{q}}_{-}(s) \overline{\mathbf{K}}_{0}(y) d y\right) \\
\mathbf{E}\left[e^{-s \tau^{+}(0)}, \gamma_{+}(0)>z, \tau^{+}(0)<\infty\right]=s^{-1} \widetilde{\mathbf{P}}^{0}(s) \mathbf{C} \int_{z}^{\infty} e^{-y \check{\mathbf{R}}_{c}(s)} \mathbf{P}_{s}^{-1} \check{\mathbf{q}}_{-}(s) \overline{\mathbf{K}}_{0}(y) d y, \\
\mathbf{E}\left[e^{-s \tau^{+}(0)}, \gamma_{0}^{+}>z, \tau^{+}(0)<\infty\right] \\
=s^{-1} \widetilde{\mathbf{P}}^{0}(s)\left(\overline{\mathbf{K}}_{0}(z)+\mathbf{C}\left(\check{\mathbf{R}}_{c}(s)\right)^{-1} \int_{z}^{\infty}\left(\mathbf{I}-e^{-y \check{\mathbf{R}}_{c}(s)}\right) \mathbf{P}_{s}^{-1} \check{\mathbf{q}}_{-}(s) d \mathbf{K}_{0}(y)\right) .
\end{gathered}
$$

Proof. Relation (3) implies that

$$
\mathbf{V}(s, x, u, v, \mu)=\overline{\mathbf{P}}_{+}(s, x) \mathbf{P}_{s}^{-1}+s^{-1} \int_{0}^{x} d \mathbf{P}_{+}(s, y) \mathbf{P}_{s}^{-1} \overline{\mathbf{G}}_{+}(s, x-y, u, v, \mu),
$$

where

$$
\overline{\mathbf{G}}_{+}(s, x, u, v, \mu)=\mathbf{G}_{+}(s, x, u, v, \mu)-\mathbf{G}_{+}(s, x, 0,0,0) .
$$


Since

$$
\begin{aligned}
\mathbf{V}_{k}(s, x, u) & =\mathbf{E}\left[e^{-s \tau^{+}(x)-u \gamma_{k}(x)}, \tau^{+}(x)<\infty\right] \\
& =\overline{\mathbf{P}}_{+}(s, x) \mathbf{P}_{s}^{-1}-u \int_{0}^{\infty} e^{-u z} \mathbf{E}\left[e^{-s \tau^{+}(x)}, \gamma_{k}(x)>z, \tau^{+}(x)<\infty\right] d z
\end{aligned}
$$

for $k=1,2,3$, equality (7) yields

$$
\begin{gathered}
\int_{0}^{\infty} e^{-u z} \mathbf{E}\left[e^{-s \tau^{+}(x)}, \gamma_{k}(x)>z, \tau^{+}(x)<\infty\right] d z \\
=-\frac{1}{s u} \int_{-0}^{x} d \mathbf{P}_{+}(s, y) \mathbf{P}_{s}^{-1} \overline{\mathbf{G}}_{k}(s, x-y, u)
\end{gathered}
$$

where

$$
\begin{gathered}
\overline{\mathbf{G}}_{k}(s, x, u)=\check{\mathbf{p}}_{-}(s) \overline{\mathbf{W}}_{k}(x, u)+\int_{-\infty}^{0-} d \mathbf{P}^{-}(s, y) \overline{\mathbf{W}}_{k}(x-y, u), \\
\overline{\mathbf{W}}_{1}(x, u)=\int_{x}^{\infty}\left(e^{u(x-z)}-\mathbf{I}\right) d \mathbf{K}_{0}(z), \quad \overline{\mathbf{W}}_{2}(x, u)=\left(e^{-u x}-\mathbf{I}\right) \overline{\mathbf{K}}_{0}(x), \\
\overline{\mathbf{W}}_{3}(x, u)=\int_{x}^{\infty}\left(e^{-u z}-\mathbf{I}\right) d \mathbf{K}_{0}(z) .
\end{gathered}
$$

Passing to the limit as $x \rightarrow 0$ we obtain from equality (8) that

$$
\int_{0}^{\infty} e^{-u z} \mathbf{E}\left[e^{-s \tau^{+}(0)}, \gamma_{k}(0)>z, \tau^{+}(0)<\infty\right] d z=-\frac{1}{s u} \mathbf{p}_{+}(s) \mathbf{P}_{s}^{-1} \overline{\mathbf{G}}_{k}(s, 0, u)
$$

Considering relation (9) for $x=0$ and taking into account equality (10) we get

$$
\begin{aligned}
& \overline{\mathbf{G}}_{1}(s, 0, u)=-u \check{\mathbf{p}}-(s)\left(\int_{0}^{\infty} e^{-u z} \overline{\mathbf{K}}_{0}(z) d z\right. \\
& \left.\quad+\mathbf{C} \int_{0}^{\infty} e^{-u z} \int_{z}^{\infty} e^{(z-y) \check{\mathbf{R}}_{c}(s)} \mathbf{P}_{s}^{-1} \check{\mathbf{q}}_{-}(s) \overline{\mathbf{K}}_{0}(y) d y d z\right), \\
& \overline{\mathbf{G}}_{2}(s, 0, u)=-u \check{\mathbf{p}}-(s) \mathbf{C} \int_{0}^{\infty} e^{-u z} \int_{z}^{\infty} e^{-y \check{\mathbf{R}}_{c}(s)} \mathbf{P}_{s}^{-1} \check{\mathbf{q}}_{-}(s) \overline{\mathbf{K}}_{0}(y) d y d z \\
& \overline{\mathbf{G}}_{3}(s, 0, u)=-u \check{\mathbf{p}}-(s)\left(\int_{0}^{\infty} e^{-u z} \overline{\mathbf{K}}_{0}(z) d z\right. \\
& \quad+\mathbf{C}\left(\check{\mathbf{R}}_{c}(s)\right)^{-1} \\
& \left.\quad \times \int_{0}^{\infty} e^{-u z} \int_{z}^{\infty}\left(\mathbf{I}-e^{-y \check{\mathbf{R}}_{c}(s)}\right) \mathbf{P}_{s}^{-1} \check{\mathbf{q}}_{-}(s) d \mathbf{K}_{0}(y) d z\right) .
\end{aligned}
$$

Substituting the above expressions for $\overline{\mathbf{G}}_{k}(s, 0, u)$ into (11) and inverting with respect to $u$ we prove (6) .

Consider some corollaries of Theorem 2.3. We also apply some of results of [2]; namely, we need an analog of the Pollachek-Khinchine formula and two-sided Lundberg inequality. Assume that $\chi_{k r}=0, k, r=1, \ldots, m$. Almost semicontinuous processes satisfying these assumptions can be treated as surplus risk processes with random premiums in a Markov environment.

Let $\zeta^{*}$ be the moment of the first jump of the process $\xi(t)$. Then

$$
\zeta_{k r}^{*} \doteq \begin{cases}\zeta_{k}+\zeta_{j r}^{*}, & \zeta_{k}^{\prime}>\zeta_{k}, x\left(\zeta_{k}\right)=j \\ \zeta_{k}^{\prime}, & \zeta_{k}^{\prime}<\zeta_{k}\end{cases}
$$


(see [1, p. 42]), where the indices $k r$ mean that $x\left(\zeta^{*}\right)=r, x(0)=k, k, r=1, \ldots, m$. Taking into account the definition of the process $Z(t)$, the latter relations imply that

$$
\begin{aligned}
\mathrm{E} e^{-s \zeta_{k r}^{*}} & =\mathrm{E}\left[e^{-s \zeta^{*}}, x\left(\zeta^{*}\right)=r / x(0)=k\right] \\
& =\mathrm{E}\left[e^{-s \zeta_{k}^{\prime}}, \zeta_{k}^{\prime}<\zeta_{k}\right] \delta_{k r}+\sum_{j=1}^{m} \mathrm{E}\left[e^{-s \zeta_{j r}^{*}+\zeta_{k}}, \zeta_{k}^{\prime}>\zeta_{k}, x\left(\zeta_{k}\right)=j\right] \\
& =\int_{0}^{\infty} \lambda_{k} e^{-s y} e^{-\lambda_{k} y} e^{-\nu_{k} y} d y \delta_{k r}+\sum_{j=1}^{m} \int_{0}^{\infty} e^{-s y} \nu_{k} e^{-\nu_{k} y} e^{-\lambda_{k} y} \mathrm{E} e^{-s \zeta_{j r}^{*}} p_{k j} d y \\
& =\lambda_{k}\left(s+\lambda_{k}+\nu_{k}\right)^{-1} \delta_{k r}+\sum_{j=1}^{m} \nu_{k}\left(s+\nu_{k}+\lambda_{k}\right)^{-1} p_{k j} \mathrm{E} e^{-s \zeta_{j r}^{*}}
\end{aligned}
$$

(see [1, p. 64]). The latter equality can be rewritten in the matrix form as follows:

$$
\mathbf{E} e^{-s \zeta^{*}}=\boldsymbol{\Lambda}(s \mathbf{I}+\boldsymbol{\Lambda}+\mathbf{N})^{-1}+(s \mathbf{I}+\boldsymbol{\Lambda}+\mathbf{N})^{-1} \mathbf{N P} \mathbf{E} e^{-s \zeta^{*}} .
$$

This implies the following representation for the moment generating function of the first jump moment:

$$
\mathbf{E} e^{-s \zeta^{*}}=(s \mathbf{I}+\boldsymbol{\Lambda}-\mathbf{Q})^{-1} \boldsymbol{\Lambda} .
$$

Since $\widetilde{\mathbf{P}}^{0}(s)=\left(\mathbf{I}-\mathbf{E} e^{-s \zeta^{*}}\right) \mathbf{P}_{s}$, we obtain

$$
\lim _{s \rightarrow 0} s^{-1} \widetilde{\mathbf{P}}^{0}(s)=(\boldsymbol{\Lambda}-\mathbf{Q})^{-1}=\left\|\mathrm{P}\left\{x\left(\zeta^{*}\right)=r / x(0)=k\right\}\right\| \boldsymbol{\Lambda}^{-1} .
$$

Let

$$
m_{1}^{0}=\sum_{k=1}^{m} \pi_{k} \int_{R} x \lambda_{k} d F_{k}^{0}(x) .
$$

Corollary 1. If $m_{1}^{0}<0$, then

$$
1-\psi_{i}(u)=\mathrm{P}_{i}\left\{\xi^{+} \leq u\right\}=\mathbf{e}_{i}^{\prime} \sum_{n=0}^{\infty} \mathbf{G}_{+}^{* n}(u)(\mathbf{I}-\|\mathbf{G}\|) \mathbf{e},
$$

where

$$
\begin{aligned}
& \mathbf{G}_{+}(y, \infty)=\mathbf{P}\left\{\gamma^{+}(0)>y, \tau^{+}(0)<\infty\right\} \\
& \quad=(\boldsymbol{\Lambda}-\mathbf{Q})^{-1}\left(\boldsymbol{\Lambda} \overline{\mathbf{F}}_{0}(y)+\mathbf{C} \int_{-\infty}^{0} e^{\check{\mathbf{R}}_{-}(0) \mathbf{C} x}\left(\mathbf{I}-\check{\mathbf{R}}_{-}(0)\right) \boldsymbol{\Lambda} \overline{\mathbf{F}}_{0}(y-x) d x\right), \\
& \|\mathbf{G}\|=\int_{0}^{\infty} \mathbf{G}_{+}(d x), \quad \mathbf{e}=(1, \ldots, 1)^{\prime}, \quad \mathbf{e}_{i}^{\prime}=(0, \ldots, i, \ldots, 0) .
\end{aligned}
$$

Proof. In fact, equality (12) is known (see the proof of Proposition 2.2 in [2]). Equality (13) follows from the first equality in (6) by passing to the limit as $s \rightarrow 0$.

Let $k(r)$ be the real eigenvalue whose real part is maximal among eigenvalues of the matrix $\mathbf{K}(r)=\mathbf{\Psi}(-\imath r)$ (that is, $k(r)$ is the Perron root of the matrix $\mathbf{K})$. Let $\gamma>0$ be a solution of the equation $k(r)=0$ and let $\boldsymbol{\nu}=\left(\nu_{1}, \ldots, \nu_{m}\right)$ and $\mathbf{h}=\left(h_{1}, \ldots, h_{m}\right)^{\prime}$ be the left and right eigenvectors of the matrix $\mathbf{K}(\gamma)$ such that their coordinates are positive and $\boldsymbol{\nu} \mathbf{h}=1$. Put

$$
C_{+}=\max _{j \in E^{\prime}} \frac{1}{h_{j}} \sup _{x \geq 0} \frac{\overline{F_{j}^{0}}(x)}{\int_{x}^{\infty} e^{\gamma(y-x)} F_{j}^{0}(d y)}, \quad C_{-}=\min _{j \in E^{\prime}} \frac{1}{h_{j}} \inf _{x \geq 0} \frac{\overline{F_{j}^{0}}(x)}{\int_{x}^{\infty} e^{\gamma(y-x)} F_{j}^{0}(d y)} .
$$


Corollary 2. If $m_{1}^{0}<0$, then

$$
C_{-} h_{i} e^{-\gamma u} \leq \psi_{i}(u) \leq C_{+} h_{i} e^{-\gamma u}
$$

for all $i \in E^{\prime}$ and $u \geq 0$.

Proof. The proof of the corollary is similar to that of Theorem 3.11 in [2].

Example. Let $Z(t)=\{\xi(t), x(t)\}$ be a process defined on the Markov chain $x(t)$ whose infinitesimal matrix is $\mathbf{Q}=\left(\begin{array}{cc}-1 & 1 \\ 1 & -1\end{array}\right)$.

Assume that $\chi_{k r}=0, k, r=1,2$. We also assume that the component $\xi(t)$ given $x(t)=i$ is represented as follows:

$$
\xi_{i}(t)=S_{i}(t)-S_{i}^{\prime}(t)=\sum_{k \leq \nu_{i}^{\prime}(t)} \eta_{k}^{i}-\sum_{k \leq \nu_{i}(t)} \xi_{k}^{i}, \quad i=1,2,
$$

where $S_{i}(t)$ and $S_{i}^{\prime}(t)$ are generalized Poisson processes with positive jumps $\xi_{k}^{i}>0$ and $\eta_{k}^{i}>0$ and parameters $\lambda_{i}$ and $\lambda_{i}^{\prime}=1$, respectively. Furthermore let

$$
\mathrm{P}\left\{\xi_{k}^{i}>x\right\}=e^{-c_{i} x}, \quad \frac{\partial}{\partial x} \mathrm{P}\left\{\eta_{k}^{i}<x\right\}=\delta_{i}{ }^{2} x e^{-\delta_{i} x}, \quad x \geq 0, i=1,2 .
$$

Consider an auxiliary process $Z_{1}(t)=\left\{\xi_{1}(t), x(t)\right\}=\{-\xi(t), x(t)\}$ whose cumulant is given by

$$
\boldsymbol{\Psi}_{1}(-\imath r)=\left(\begin{array}{cc}
\frac{-3 r^{3}+\left(2 c_{1}-6 \delta_{1}\right) r^{2}+\left(4 c_{1} \delta_{1}-2 \delta_{1}^{2}\right) r+c_{1} \delta_{1}}{\left(r-c_{1}\right)\left(r+\delta_{1}\right)^{2}} & 1 \\
1 & \frac{-3 r^{3}+\left(2 c_{2}-6 \delta_{2}\right) r^{2}+\left(4 c_{2} \delta_{2}-2 \delta_{2}^{2}\right) r+c_{2} \delta_{2}}{\left(r-c_{2}\right)\left(r+\delta_{2}\right)^{2}}
\end{array}\right) .
$$

Then the stationary distribution is $\boldsymbol{\pi}=\left(\frac{1}{2}, \frac{1}{2}\right)$. If $c_{1}=\frac{1}{3}, c_{2}=\frac{1}{2}, \delta_{1}=2$, and $\delta_{2}=1$, then $m_{1}^{0}=1>0$. According to Theorem 3 of [4], $\xi_{1}^{-}$has a nondegenerate distribution. Consider the matrix

$$
\mathbf{G}(r):=r \mathbf{\Psi}_{1}^{-1}(-\imath r)(\mathbf{C}-r \mathbf{I})^{-1}=\frac{1}{D(r)}\left(\begin{array}{ll}
g_{11}(r) & g_{12}(r) \\
g_{21}(r) & g_{22}(r)
\end{array}\right),
$$

where $D(r)=48 r^{5}+263 r^{4}+387 r^{3}+114 r^{2}-51 r-8, g_{11}(r)=3(r+2)^{2}\left(6 r^{3}+10 r^{2}-1\right)$, $g_{12}(r)=2(r+1)^{2}(r+2)^{2}(3 r-1), g_{21}(r)=3(r+1)^{2}(r+2)^{2}(2 r-1)$, and

$$
g_{22}(r)=2(r+1)^{2}\left(9 r^{3}+34 r^{2}+16 r-4\right) .
$$

Since $D(r)$ has four negative roots,

$$
-\rho_{1}=-3.25672, \quad-\rho_{2}=-1.59682, \quad-\rho_{3}=-0.794382, \quad-\rho_{4}=-0.133485,
$$

and one positive root $r_{0}=0.30224$, the entries of the matrix $\mathbf{G}(r)$ are such that

$$
G_{i j}(r)=C_{i j}^{0}+\frac{C_{i j}^{1}}{r+\rho_{1}}+\frac{C_{i j}^{2}}{r+\rho_{2}}+\frac{C_{i j}^{3}}{r+\rho_{3}}+\frac{C_{i j}^{4}}{r+\rho_{4}}+\frac{C_{i j}^{5}}{r-r_{0}} .
$$

The projection for functions of the form

$$
\mathbf{G}(r)=\mathbf{C}_{0}+\int_{-\infty}^{\infty} e^{r x} \mathbf{g}(x) d x
$$

is defined by

$$
[\mathbf{G}(r)]^{-}=\int_{-\infty}^{0} e^{r x} \mathbf{g}(x) d x
$$

Considering the projection we get

$$
G_{i j}^{-}(r)=\left[G_{i j}(r)\right]^{-}=\frac{C_{i j}^{1}}{r+\rho_{1}}+\frac{C_{i j}^{2}}{r+\rho_{2}}+\frac{C_{i j}^{3}}{r+\rho_{3}}+\frac{C_{i j}^{4}}{r+\rho_{4}} .
$$


Since

$$
\check{\mathbf{R}}_{+}=\left(\mathbf{G}^{-}(0)+(\boldsymbol{\Lambda}-\mathbf{Q})^{-1}\right)^{-1} \mathbf{P}_{0}=\left(\begin{array}{cc}
0.22 & 0.22 \\
0.17 & 0.17
\end{array}\right)
$$

Theorem 3 of [4] implies that

$$
\begin{aligned}
\mathrm{E}_{i}\left[e^{r \xi_{1}^{-}}, \xi_{1}^{-}<0\right] & =\mathbf{E}\left[e^{r \xi_{1}^{-}}, \xi_{1}^{-}<0\right] \cdot \mathbf{e}=[\mathbf{G}(r)]^{-} \check{\mathbf{R}}_{+} \cdot \mathbf{e} \\
& =\frac{A_{i}^{1}}{r+\rho_{1}}+\frac{A_{i}^{2}}{r+\rho_{2}}+\frac{A_{i}^{3}}{r+\rho_{3}}+\frac{A_{i}^{4}}{r+\rho_{4}}, \quad i=1,2,
\end{aligned}
$$

where $\mathbf{e}=(1,1)^{\prime}$. Inverting with respect to $r$ we evaluate the distribution of $\xi_{1}^{-}$as follows:

$$
\mathrm{P}_{i}\left\{\xi_{1}^{-}<x\right\}=\sum_{k \leq 4} \frac{A_{i}^{k}}{\rho_{k}} e^{\rho_{k} x}, \quad x<0 .
$$

Therefore

$$
\begin{aligned}
\psi_{1}(u) & =\mathrm{P}_{1}\left\{\xi^{+}>u\right\}=\mathrm{P}_{1}\left\{\xi_{1}^{-}<-u\right\} \\
& \approx-0.04 e^{-3.26 u}+0.001 e^{-1.6 u}+0.079 e^{-0.79 u}+0.75 e^{-0.13 u} \\
\psi_{2}(u) & =\mathrm{P}_{2}\left\{\xi^{+}>u\right\}=\mathrm{P}_{2}\left\{\xi_{1}^{-}<-u\right\} \\
& \approx-0.01 e^{-3.26 u}-0.016 e^{-1.6 u}+0.004 e^{-0.79 u}+0.85 e^{-0.13 u}
\end{aligned}
$$

On the other hand, one can use bounds (14):

$$
\begin{aligned}
& 0.665 e^{-0.13 u} \leq \psi_{1}(u) \leq 0.935 e^{-0.13 u}, \\
& 0.757 e^{-0.13 u} \leq \psi_{2}(u) \leq 1.064 e^{-0.13 u} .
\end{aligned}
$$

Passing to the limit in (6) as $s \rightarrow 0$ we deduce that

$$
\begin{aligned}
& \mathbf{P}\left\{\gamma^{+}(0)>z, \tau^{+}(0)<\infty\right\} \approx\left(\begin{array}{cc}
e^{-2 z}(0.48+0.86 z) & e^{-z}(0.31+0.22 z) \\
e^{-2 z}(0.21+0.34 z) & e^{-z}(0.61+0.49 z)
\end{array}\right), \\
& \mathbf{P}\left\{\gamma_{+}(0)>z, \tau^{+}(0)<\infty\right\} \approx\left(\begin{array}{cc}
0.1 e^{-2 z}(1+z) & e^{-z}(0.2+0.1 z) \\
0.09 e^{-2 z}(1+z) & e^{-z}(0.18+0.09 z)
\end{array}\right) \\
&+\left(\begin{array}{cc}
e^{-2.3 z}(0.0016+0.002 z) & -e^{-1.3 z}(0.02+0.013 z) \\
-e^{-2.3 z}(0.004+0.004 z) & e^{-1.3 z}(0.05+0.03 z)
\end{array}\right), \\
& \mathbf{P}\left\{\gamma_{0}^{+}>z, \tau^{+}(0)<\infty\right\} \approx\left(\begin{array}{cc}
e^{-2 z}\left(0.49+0.97 z+0.2 z^{2}\right) & e^{-z}\left(0.3(1+z)+0.1 z^{2}\right) \\
e^{-2 z}\left(0.2+0.4 z+0.18 z^{2}\right) & e^{-z}\left(0.7(1+z)+0.09 z^{2}\right)
\end{array}\right) \\
&+\left(\begin{array}{cc}
-e^{-2.3 z}(0.005+0.01 z) & e^{-1.3 z}(0.03+0.04 z) \\
e^{-2.3 z}(0.01+0.03 z) & -e^{-1.3 z}(0.075+0.1 z)
\end{array}\right) .
\end{aligned}
$$

\section{BIBLIOGRAPHY}

1. D. V. Gusak, Boundary Problems for Processes with Independent Increments on Finite Markov Chains and for Semi-Markov Processes, Institute of Mathematics, National Academy of Sciences of Ukraine, Kyiv, 1998. (Ukrainian) MR.1710395 (2000m:60050)

2. S. Asmussen, Ruin Probabilities, World Scientific, Singapore, 2000. MR 1794582 (2001m:62119)

3. D. V. Gusak, The distribution of extrema for risk processes on a finite Markov chain, Theory Stoch. Process. 7(23) (2001), no. 1-2, 109-120. 
4. D. V. Gusak and E. V. Karnaukh, Matrix factorization identity for almost semi-continuous processes on a Markov chain, Theory Stoch. Process. 11(27) (2005), no. 1-2, 40-47. MR2327445 (2008e:60314)

5. V. S. Korolyuk and A. F. Turbin, Semi-Markov Processes and their Applications, "Naukova dumka", Kiev, 1976. (Russian) MR0420902 (54:8913)

Department of Probability Theory and Mathematical Statistics, Faculty for Mechanics and Mathematics, National Taras Shevchenko University, Academician Glushkov Avenue 6, KYIV 03127, UKRAINE

E-mail address: kveugene@univ.kiev.ua

Received 31/OCT/2005

Translated by N. SEMENOV 